\title{
A Multicriteria Optimization for Flight Route Networks in Large-Scale Airlines Using Intelligent Spatial Information
}

\author{
Mostafa Borhani*, Kamal Akbari, Aliakbar Matkan, Mohammad Tanasan \\ Shahid Beheshti University (Iran)
}

Received 30 May 2019 | Accepted 14 November 2019 | Published 25 November 2019

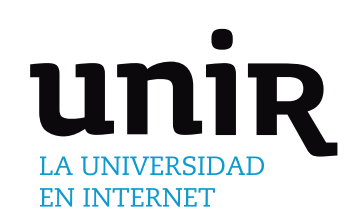

KEYWORDS

Air route network optimization, one of the airspace planning challenges, effectively manages airspace resources toward increasing airspace capacity and reducing air traffic congestion. In this paper, the structure of the flight network in air transport is analyzed with a multi-objective genetic algorithm regarding Geographic Information System (GIS) which is used to optimize this Iran airlines topology to reduce the number of airways and the aggregation of passengers in aviation industries organization and also to reduce changes in airways and the travel time for travelers. The proposed model of this study is based on the combination of two topologies point-to-point and Hub-and-spoke - with multiple goals for causing a decrease in airways and travel length per passenger and also to reach the minimum number of air stops per passenger. The proposed Multi-objective Genetic Algorithm (MOGA) is tested and assessed in data of the Iran airlines industry in 2018, as an example to real-world applications, to design Iran airline topology. MOGA is proven to be effective in general to solve a network-wide flight trajectory planning. Using the combination of point-to-point and Hub-and-spoke topologies can improve the performance of the MOGA algorithm. Based on Iran airline traffic patterns in 2018 , the proposed model successfully decreased $50.8 \%$ of air routes (184 air routes) compared to the current situations while the average travel length and the average changes in routes were increased up to $13.8 \%$ (about 100 kilometers) and up to $18 \%$, respectively. The proposed algorithm also suggests that the current air routes of Iran can be decreased up to $24.7 \%$ ( 89 airways) if the travel length and the number of changes increase up to $4.5 \%$ (32 kilometers) and 5\%, respectively. Two intermediate airports were supposed for these experiments. The computational results show the potential benefits of the proposed model and the advantage of the algorithm. The structure of the flight network in air transport can significantly reduce operational cost while ensuring the operation safety. According to the results, this intelligent multi-object optimization model would be able to be successfully used for a precise design and efficient optimization of existing and new airline topologies.

\author{
Multi-Objective \\ Optimization, Genetic \\ Algorithm, Airway \\ Topology, GIS, Artificial \\ Intelligence, Non- \\ Dominated Sorting \\ Genetic Algorithm II \\ (NSGA-II).
}

\section{INTRODUCTION}

\begin{abstract}
A IRLINE network topology consists of many different and wideranging parameters such as travel length, the number of airways, and changes in routes, as well as some rules and constraints like rerouting, flight-level changes, and a limited airport capacity [1]. Air route network optimization as a multipurpose optimization of an indescribable type or the so-called Non-deterministic Polynomialtime hard (NP-Hard) [2] [3] [4] should be considered in hub network design problem [5]. This paper suggests employing the capabilities of Geographic Information Systems (GIS) as powerful tools regarding the management, analysis, and offer of spatial information for designing topology of airline route networks [6]. Moreover, a Nondominated sorting genetic algorithm II (NSGA-II) [7] as a multipurpose optimization algorithm is implemented in this study. The Multi-objective Genetic Algorithm (MOGA) is exploited because the obtained Pareto Front of MOGA is very accurate and fast [8]. MOGA as one type of well-known and robust genetic optimization algorithms
\end{abstract}

\footnotetext{
* Corresponding author.

E-mail address: mo_borhani@sbu.ac.ir
}

is one of the most popular multicriteria optimization algorithms which is also a potent technique for solving problems concerning research and optimization in the real world. Using the Pareto ranking and the crowding distance in the ranking of the individuals encourage various applications especially, those regarding GIS to exploit this algorithm because of the absence of additional parameters in MOGA as well as its high computational speed [7].

GIS knowledge in spatial modeling and simulation especially with soft-computing methods [9] such as multi-objective genetic algorithm [10] can be used in designing airways topology. Intelligent algorithms such as multi-objective optimization algorithms could help GIS dealing with matters concerning spatial decision-making [11]. Multi-objective evolutionary algorithms take a more evolutionary approach to learning than traditional artificial intelligence (AI) [12] and it is usually facing a vast amount of data and processing. Typically, the environment that requires optimization has different objectives and complications, so, a fast algorithm could be a convenient option [13] [14].

Modelling the air transport with complex networks [15] is one of the essential issues in the transport geography and GIS, which can be optimized by methods for generating non-dominated solutions (the weighting, and constraint methods), the distance-based methods (such as compromise programming, goal programming, and reference point 
methods), and interactive methods. By choosing a proper topology for air routes in GIS, airlines can save costs and reduce risk in the air industry [5]. Moreover, aviation industries could offer cheaper yet high-quality services which will enhance the travelers' satisfaction and finally boost the air industry.

There have been numerous studies about the designing of airline topology and its optimization. O'Kelly introduced a hub locating using a single-center air network that was modeled using a numerical scheduling method [2]. His results demonstrated that this matter belongs to NP-hard issues and it requires a counting method based on innovative algorithms to be solved [2]. He assumed only one hub. Park and Sohn tried to explain a two-hub locating through linear planning [2]. They concluded that when there are two hubs, we can use linear planning [16]. Marianova and Serra [17] worked on modeling hub locating in airline networks by considering swarm at intermediate airports in the presence of $\mathrm{M} / \mathrm{D} / \mathrm{c}$ queue. In their model, they used an exploratory method based on forbidden search and implemented it on American Airways Dataset (CAB) [17]. Wagner performed clustering through a genetic algorithm and banned search to solve incapacitated multiple allocation hub location problems (UHLP-M) [18]. Mohammadi et al. considered swarm at hubs once all its functionality has been lost and figured it out through a multi-server queueing Model, M/M/c queue system (or Erlang-C Model) [19]. Alumur et al. used mixed-integer programming for locating intermediate airports at multiple levels for Turkey and studied sensitivity analysis and performed a more accurate analysis of hub locating [20]. Taghipourian et al. examined a hub network in the event of impairment. They considered some hubs as virtual hubs to replace them once the central hub was closed. Therefore, they employed fuzzy correct linear programming [21]. Chiu and Yang worked on the designing of airlines in the Hub-and-spoke system with a random request for intermediate airports and the impact of the swarm on them. They formulated the issue with a two-step randomized programming and used China and Taiwan's airline dataset to test and assess the sensitivity of the model [22]. Harshavardhan and Krishnan proposed a framework of genetic algorithms, which use multi-level hierarchies to solve an optimization problem by searching over the space of simpler objective functions with capacities to model and solve hub locating. They used a heuristic approach based on two genetic algorithms and a hierarchical adjacency search. Their results suggested the high influence of the hierarchical genetic algorithms [23]. Using a multi-objective genetic algorithm based on game theory as well as a hybrid simulated annealing algorithm are some of the comparative optimization approaches for the NSGA-II in the bi-objective hierarchical hub location problem [24]. By proposing the combination of point-to-point and Hub-andspoke topologies in the present paper, MOGA simultaneously finds a representative set of Pareto optimal solutions and convergences faster than its comparator algorithms for air route network optimization.

The rest of this paper is organized as follows: First, we give an overview of the point-to-point and Hub-and-spoke topologies in section II followed by the proposed research question, which aimed to optimize three goals (reducing the number of airways, the number of air stops and the flight length) simultaneously. In section III, an optimal model for airline topologies based on a multi-objective genetic algorithm has been proposed and detailed. Three object functions are introduced and the intelligent multi-object optimization model is presented. The implementation of the proposed model by genetic algorithm and the dataset specifications are described in detail in section IV, followed by the simulation results for the proposed MOGA algorithm for Iran airline topology. Then, the discussions about the research results, especially assessing the proposed model and the proposed model repeatability test are reported. At the end of the conclusions, the section states the main deductions of using a multi-objective genetic algorithm for Iran airline network optimization.

\section{Point-to-Point And Hub-And-Spoke Topologies}

Air route network optimization was motivated by the purpose of defining the most effective structural properties for a given air transport network, in terms of the number of airways and the aggregation of passengers in aviation industries organization, and also the changes in airways and the travel time for travelers. There are two major groups of air transportation networks:

- Point-to-point: in this construction of the air traffic network, each justified connection pair of airports in the network is served by direct connections to all passengers with different aircraft. The number of connections and required aircrafts is about the square of the number of airports for a fully point-to-point connected network. An example of a point-to-point architecture is depicted in Fig. 1.

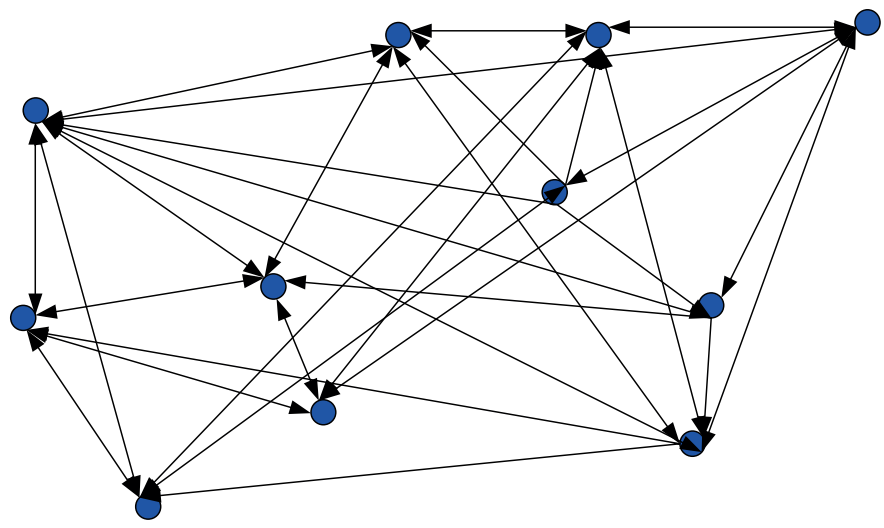

Fig. 1. The point-to-point architecture of air routes.

- Hub-and-spoke: Hub-and-spoke topology is one of the proper topologies in airway networks [25]. In this topology, instead of a direct flight between all airports, some of the terminals are considered as intermediate airports (hub airports) and other airports (spoke airports) are only connected to the intermediate airports (Hub-and-spoke architecture, Fig. 2). In this topology, instead of a direct flight between departure and destination airports, at first, all the passengers fly together from the same departures to different destinations to one of these intermediate airports. Then, they will be gone to the different departures from an intermediate airport. In the meantime, if necessary, flights between the intermediate airports will be made. In this topology with the lowest number of air routes, most airline coverage can be achieved [4].

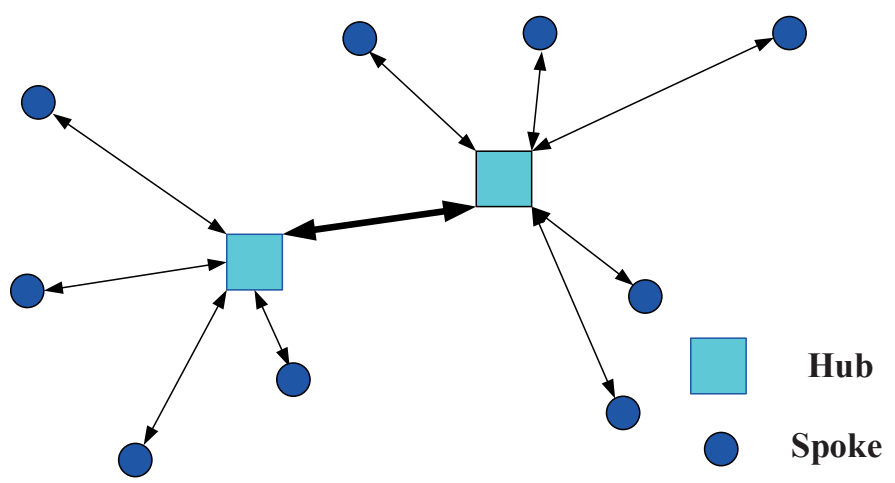

Fig. 2. The Hub-and-spoke architecture of air routes.

The point-to-point architecture of air routes, for a complete network of $N$ airports, so that all airports can be connected (point-topoint topology), $N(N-1)$ air routes are needed. All $N(N-1)$ airlines do not have similar air traffic and on the other hand, the high number 
of air routes increases the flight cost. To decrease the number of air routes, the hub-and-spoke topology can be used. Through the following equation, the total number of needed airlines in this topology with $M$ intermediate airports from $N$ available airports can be calculated:

$$
\text { Number of Airlines }=2(N-M)+\frac{M(M-1)}{2}
$$

The number of reduced flights in comparison with the direct flights from all airports can be calculated from the following equation:

$$
\begin{aligned}
\text { Reduction of Airlines } & =N(N-1)-2(N-M)+\frac{M(M-1)}{2} \\
& =N(N-3)+\frac{M(M-5)}{2}
\end{aligned}
$$

For instance, with 60 airports $(N=60)$ in the point-to-point topology, $60 * 59=3540$ airlines are needed, however, by considering an intermediate airport $(M=1)$ in the Hub-and-spoke topology, the number of airways is decreased to $2(60-1)+\frac{1(1-1)}{2}=118$. Of course, you have to think that in the Hub-and-spoke topology, the number of air stops as well as the flight length will increase but due to the aggregation of passengers, the airplanes will carry more passengers [26]. The USA airline with two central airports located in Atlanta and Chicago or even Dubai airport as an intermediate airport in the Middle East are two examples of the Hub-and-spoke topology. This study solves the problem of optimization of all mentioned goals (reducing the number of airways, the number of air stops and the flight length) simultaneously by proposing a solution based on the combination of two topologies - point-to-point and Hub-and-spoke - with MOGA.

The optimization of topology in an airway network reduces airfare rates and boosts the flight industry. Studies have shown that in solving the problem of hub locating, first researchers concerned about parameter modeling, and then the focus has been shifted on optimal Modeling for hub problem solving and recently, the large-scale optimization issues and algorithm selection approaches have been addressed. Also, these studies acknowledged the multi-dimensional and NP-hard features of hub locating as presented in this paper.

\section{The Proposed Topology of Airlines}

The primary purpose of this paper is to design an optimal model for airline topologies based on a multi-objective genetic algorithm. Since the point-to-point topology and Hub-and-spoke topology have their benefits - in the point-to-point topology, the travel length is short, and the number of changes in direction is few. However, in the Hub-andspoke topology, the number of airlines will decrease - the proposed topology of this study is defined to provide a combination of both topologies. In this topology, if necessary, an airport could connect to more than one intermediate airports or another regular airport (Fig. 3). In many studies, for the allocation of a spoke to the airport, the distance criterion has been used. In these cases, each airport has been allocated to its nearest intermediate hub.

An innovative structure based on the artificial intelligence was employed for allocating the airports to an intermediate hub by this paper. In this proposed topology, at first, the matrix of the number of passengers between airports and the matrix of the distance between airports was provided. Afterward, by using the capabilities of the MOGA algorithm, an optimal topology for the airlines was determined to achieve the supposed objectives. This model has been defined in a way to create an airline topology in which, with the slightest increase in average travel length and the average changes in paths, the number of flight routes would decrease. The outcomes of this model were presented as optimal airline topologies with the same applied value. Based on the conditions, an expert opinion, and policymakers, we could implement one of these optimal topologies. On the other hand, if due to whatever reason such as adverse weather conditions, one of these intermediate airports is excluded from this topology, we could choose another one as an intermediate airport.

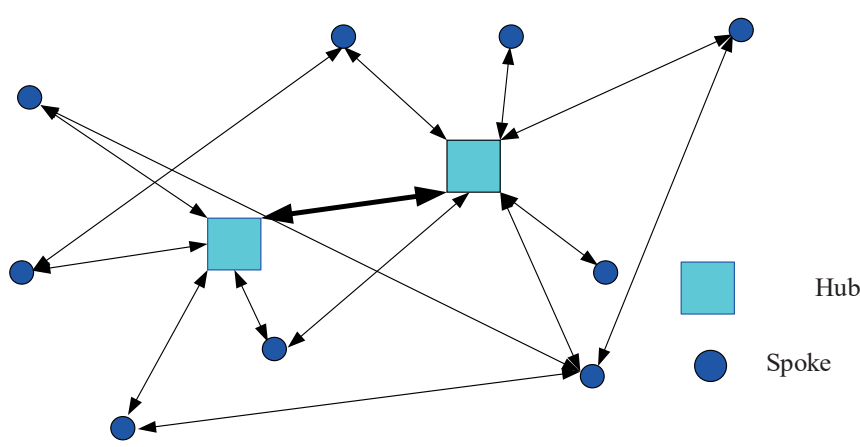

Fig. 3. The proposed combined structure of airlines (the combination of pointto-point and Hub-and-spoke architectures).

The designed model in this study comprises two main sections. One section is responsible for optimizing and finding intermediate airports, and the other section provides optimal airline topologies with the presence of the perceived intermediate airports in the first part. The flowchart of the proposed model is shown in Fig. 4.

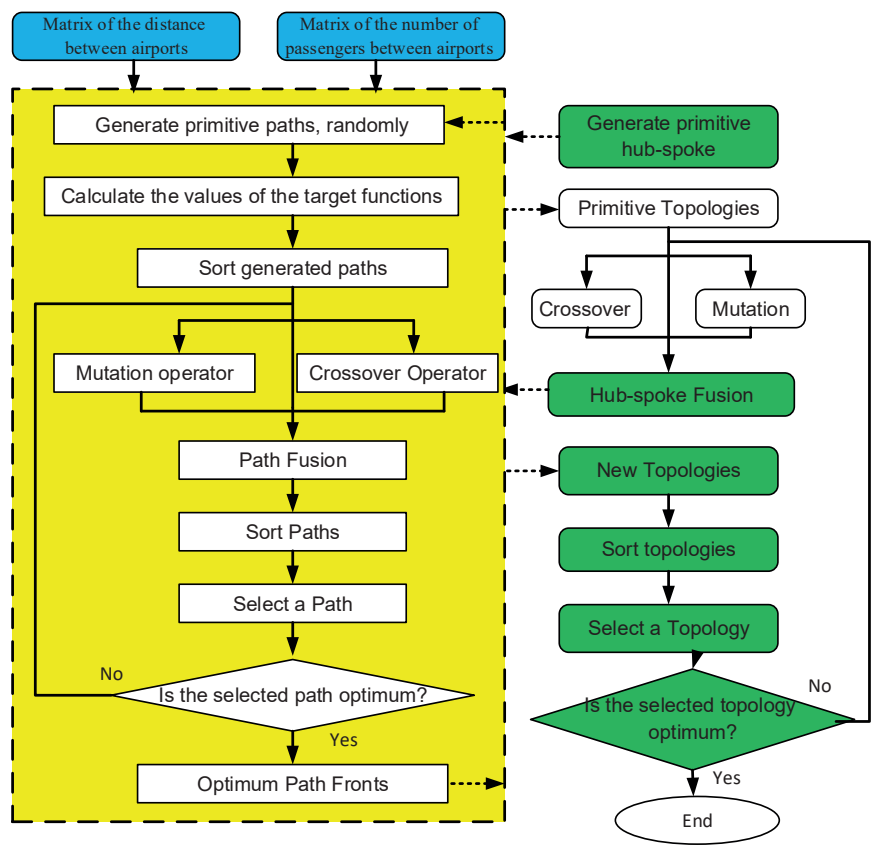

Fig. 4. The flowchart of the proposed model.

The MOGA algorithm was employed for providing optimal airline topologies in this paper. Since this model is a multi-objective one, there are multiple optimal topologies for each set of intermediate airports, which cover the outputs of optimal airline topologies. It should be noted that the amounts of each objective function for every response in the offering section of optimal topology can be calculated.

To design the airline topologies, three objective functions for simultaneously reducing the number of airlines, Object $t_{1}$, the average travel length, Object ${ }_{2}$, and the average number of air stops, Object $_{3}$, were taken into account that can be described as follows:

To design the airline topologies, three objective functions for simultaneously reducing the number of airlines, Object ${ }_{1}$, the average travel length, Object ${ }_{2}$, and the average number of air stops, Object $_{3}$, 
were taken into account that can be described as follows:

$$
\min \left(\text { Object }_{1} \text { Object }_{2,} \text { Object }_{3}\right)
$$

\section{A. First Object Function: Reducing the Number of Airlines}

The reduction of the number of airlines is one issue that can be considered while designing airline topologies. The highest number of airways is in the point-to-point system, and the lowest one is the Huband-spoke system. In this study, a combination of these two systems was employed to offer airline topologies. This target function was designed based on the following equation:

$\min \left(\right.$ Object $\left._{1}\right)=\min \left(\sum R_{S_{i} \rightarrow H}+\sum R_{H \rightarrow S_{j}}+\frac{n_{H}\left(n_{H}-1\right)}{2}+\sum R_{S_{i} \rightarrow S_{j}}\right)_{(4)}$

In the above-mentioned equation, $R_{S_{i} \rightarrow H}$ is air route from a departure airport to an intermediate airport, $R_{H \rightarrow S_{j}}$ is air route from an intermediate airport to a destination airport, $R_{S_{i} \rightarrow S_{j}}$ is a direct air route between departure and destination airports, and $n_{H}$ is the number of intermediate airports.

\section{B. Second Object Function: Reducing the Average Travel Length}

Another goal is reducing the average travel length, Object $_{2}$. This target function conflicts with the target function of reducing the number of airways, Object $_{1}$, because by reducing the number of airways, the journey time will increase. In other words, the shortest average travel length is in the point-to-point system, and the longest one is in the Hub-and-spoke system with one spoke. Following equation shows the implementing process of this target function:

$$
\min \left(\text { Object }_{2}\right)=\min \left(\frac{\sum \sum P_{i \rightarrow j}\left(D_{S_{i} \rightarrow H_{m}}+D_{H_{m} \rightarrow H_{n}}+D_{H_{n} \rightarrow S_{j}}\right)}{\sum \sum P_{i \rightarrow j}}\right)
$$

where $P_{i \rightarrow j}$ is the number of passengers from a departure airport $\left(S_{i}\right)$ to a destination airport $\left(S_{j}\right), D_{S_{i} \rightarrow H_{m}}$ is the distance between the departure airport and an intermediate airport, $D_{H_{m} \rightarrow H_{n}}$ is the distance between two intermediate airports, and $D_{H_{n} \rightarrow S_{j}}$ is the distance to a destination airport from an intermediate airport.

\section{Third Object Function: Reducing the Average Change in Route}

To increase the welfare of the passengers and reduce the waiting time at the airport, it is necessary to reduce as many changes in routes and air stops as possible, Object $_{3}$. The lowest amount of changes in directions exists in the point-to-point topology. In other words, in this topology, each passenger will use only one airline from departure to destination. Following equation displays the implementation process of this target function:

$$
\min \left(\text { Object }_{3}\right)=\min \left(\frac{\sum \sum P_{i \rightarrow j}\left(\delta_{S_{i} \rightarrow H_{m}}+\delta_{H_{m} \rightarrow H_{n}}+\delta_{H_{n} \rightarrow S_{j}}\right)}{\sum \sum P_{i \rightarrow j}}\right)
$$

In the above equation, $P_{i \rightarrow j}$ is the number of passengers from a departure airport $\left(S_{i}\right)$ to a destination airport $\left(S_{j}\right)$. Also, $H_{m}$ is an intermediate airport for departure and $H_{n}$ is an intermediate airport for a destination. In this equation, if there is a flight between two airports $\left(S_{i} \rightarrow H_{m}, H_{m} \rightarrow H_{n}\right.$ or $\left.H_{n} \rightarrow S_{j}\right)$, the number of $\delta$ equals to 1 , otherwise, 0 . It should be noted that in the two above equations, if it is supposed to be a direct flight - without an intermediate airport - from the departure airport to destination airport, $S_{i}$ will be considered equal to $H_{m}$ and $S_{i}$ equal to $H_{n}$.

Briefly, our proposed multi-object optimization problem can be formulated as a minimization of the above-mentioned objects simultaneously which has been done by MOGA in this paper.
IV. The Implementation of the Proposed Model

In this study, to solve issues regarding the design of optimal airline topologies through the MOGA algorithm, a chromosome was first designed following the type of problem and then to perform the model, crossover, and mutation genetic operators were applied, and then appropriate choices to the kind of problem were employed. The implementation process of these matters is described as follows:

A set of chromosomes is considered as a population for the genetic algorithm [27] that evolves with its operators. In the first section of the model, a chromosome was used for encoding intermediate airports.

\begin{tabular}{|c|c|c|c|c|c|c|}
\hline ID Airport & 1 & 2 & 3 & $\ldots$ & $n-1$ & $\mathrm{n}$ \\
\hline Hubs & 0 & 1 & 0 & $\ldots$ & 0 & 1 \\
\hline
\end{tabular}
This chromosome is encoded in binary, indicating whether an airport is an intermediate (code 1) or not (code 0) (Fig. 5).

Fig. 5. A chromosome identifying intermediate airports among $\mathrm{n}$ airports.

In the second section of the model, a chromosome is considered for determining the MOGA of the connections and networks among airlines. This chromosome displays airline topologies and comprises two gene sequences along the length of the airways; the first series determines an intermediate airport for the departure airport and the second one identifies an intermediate airport for the destination airport of each airway (Fig. 6). The value of the chromosome can be obtained from the first section of the model, which included the identified intermediate airports. Some zero genes in this chromosome are taken into account for situations where an intermediate airport is

\begin{tabular}{|c|c|c|c|c|c|c|c|c|}
\hline ID AirLine & 1 & 2 & 3 & 4 & $\ldots$ & $\mathrm{m}-2$ & $\mathrm{~m}-1$ & $\mathrm{~m}$ \\
\hline Hubs for Origin & 7 & 36 & 0 & 36 & $\ldots$ & 0 & 7 & 7 \\
\hline Hubs for Destination & 0 & 36 & 0 & 7 & $\cdots$ & 36 & 0 & 7 \\
\hline
\end{tabular}
not considered. If the value of a gene in two strings is zero, it will indicate that the flight between the two airports will happen directly.

Fig. 6. A chromosome for determining of connection and network of airlines with two intermediate airports coded $7 \& 36$ for $m$ airways.

One of the essential operators in MOGA algorithms is the crossover operator. It aims to explore new and especially useful areas of the research environment by replacing a part of the gene sets between the two chromosomes. In the present study, a one-point and two-point crossover operators [28] were employed. It should be noted that to create more various compounds from two parents, in the second chromosome, for each gene sequence, a crossover operator was applied separately.

The mutation operator imports new information to the population and searches in empty spaces of the problem. In this study, some genes were randomly selected to be mutated. The amount of that gene was then replaced by the values allowed for that gene.

\section{A. Area of Study and Dataset}

To test and assess the proposed model for designing Iran airline topology, the data of the Iran Airlines Industry from 2018 was taken into account. According to this data, in 2018, 18251677 passengers traveled by the air transport system with 433 airlines from 60 airports with 163407 flights. Averagely, the travel length per person was 724 kilometers, and the number of passengers per flight was 111.7. The most frequent travels were from Mehrabad Airport in Tehran. Concerning passengers' movement, Mashhad Airport was in the $2^{\text {nd }}$ place. The least amount of flights belonged to Siravan and Jask Airport with only one trip. The best airport in terms of the number of travelers per flight was 
Kerman Airport with 172.3 passengers per flight, and the worst airport in the country (disregarding airports with one trip) was Iranshahr Airport with only 38.2 passengers per flight. The most crowded airline was from Kerman Airport to Tehran Airport with 229.4 passengers per flight and from Tehran to Kerman with 227.6 passengers per flight. The most noticeable amount of passengers belonged to the airline from Mashhad to Tehran with 1726714 travelers, and the most significant amount of trips belonged to the same airline with 12468 flights.

The database of this study which was used for designing optimal airline topologies included statistical information on Iran airways. The statistics regarding the movement of passengers among airports in the country in 2018 got extracted from the analytical journal of the Ministry of Roads and Urban Development related to the Aviation Division and the locations of the country's airports in the Lambert coordinate system, as well as Google Earth application.

Those routes that had certain conditions such as the relocation of national authorities or relocating without passengers between two airports got removed from research. Therefore, the average passengers per airline was considered as 100 . Also, the number of airlines changed from 433 to 360 efficient carriers. The Iran's passengers' movement matrix was used.

Another input of the proposed model was the matrix of air distance between airports. The most accurate method for calculating the length of the airways is Euclidean relation. Hence, at first, the spatial location of airports was calculated in Lambert coordinate system, and then the following equation was used for calculating air route length.

$$
\operatorname{Distance}(A, B)=\left(\left(X_{A}-X_{B}\right)^{2}+\left(Y_{A}-Y_{B}\right)^{2}\right)^{\frac{1}{2}}
$$

In the above equation, $X_{A}$ and $Y_{A}$ are the geographical length and width of Airport $A$ (departure airport), and $X_{B}$ and $Y_{B}$ are the topographical length and breadth of Airport $B$ (destination) in Lambert coordinate system. After obtaining the distance between airports, the matrix of airport distances was provided.

\section{Simulation Results}

The output of the proposed multi-objective genetic algorithm offered multiple optimal airline topologies (a combination of point-to-point system and Hub-and-spoke system) that are equal from the optimization value and the final optimal architectures can be selected and applied based on the policies in the field of air industry and experts' opinions.

For the described dataset, one of the model outputs regarding optimal airways which includes Mehrabad and Mashhad airports as intermediate airports, is the growth of the average travel length up to $13.8 \%$ (about 100 kilometers) and also by increasing the average changes in routes up to $18 \%, 50.8 \%$ of air routes (184 air routes) will decrease compared to the current situations. Moreover, in another case with the same air intermediate, by increasing the travel length to $4.5 \%$ (32 kilometers) and the number of changes in routes to $5 \%, 24.7 \%$ of the air routes (89 airways) will decrease. Table I shows some of the topologies in model output.

The number of airways, and the average travel length for the optimal topology with two target functions is shown in Fig. 8-A. Fig. 8-B shows the number of airways and changes in routes for the proposed algorithm with two target functions. There is a reverse relationship between the target function of numbers of airways and other target functions - average travel length and the average number of changes in routes. On the other hand, this optimal topology indicates there is a direct relationship between the target function of average travel length and average changes in directions. Fig. 7 shows some of the topologies of model output.

The optimal answers to this problem have a three-dimensional space in the number of target functions. To have a better display of the answer curve, optimal solutions for target functions were offered twoto-two in a $2 \mathrm{D}$ environment (Fig. 8-A, B \& C) and the rest of the target functions were presented in a 3D environment (Fig.8-D).

According to the output results, the proposed model can offer optimal answers and is significantly better than the current situations of the country's airways. The presence of different answers with intermediate airports indicates the model's capability in providing a combined airway topology from the combination of point-to-point and Hub-and-spoke topologies (e.g., answers No. 4 \& 5 in Table I). For allocating air routes between an airport and one of the intermediate airports in the proposed model, Genetic algorithm optimization methods were employed instead of considering the nearest intermediate airport. Of course, in many cases, the nearest intermediate airport to each airport has been selected as the intermediate airport in that airport, however, not always. For example, if Mashhad airport and Isfahan airport are chosen as an intermediate airport, based on the distance, it is expected that Mashhad airport would be the intermediate airport for Iranshahr airport. However, given the results of model output, it can be seen that Isfahan airport with a more significant distance has been selected as an intermediate airport for Iranshahr airport. In the rest of this section, the model structure will be assessed.

\section{A. Assessing the Proposed Model}

In this study, the convergence plot and the path non-constrained-

Table I. Number of Optimal Airline Topologies of Model Output

\begin{tabular}{|c|c|c|c|c|c|}
\hline No. & Aviation centers & Number of airways & $\begin{array}{l}\text { Average travel } \\
\text { length }\end{array}$ & Average air stop & $\begin{array}{l}\text { Number of direct } \\
\text { flights }\end{array}$ \\
\hline 1 & - & 360 & $7 / 747$ & 1 & 362 \\
\hline 2 & Mehrabad & 115 & 897 & 1.26 & 0 \\
\hline 3 & Mehrabad - Isfahan & 115 & 870 & 1.74 & 0 \\
\hline 4 & Mehrabad - Mashhad & 139 & 882 & 1.21 & 2 \\
\hline 5 & Mehrabad - Mashhad & 178 & 851 & 1.18 & 32 \\
\hline 6 & Mehrabad - Mashhad & 271 & 780 & 1.05 & 113 \\
\hline 7 & Mehrabad - Mashhad - Shiraz - Ahvaz & 250 & 777 & 1.13 & 45 \\
\hline 8 & Mehrabad - Mashhad - Isfahan - Shiraz - Ahvaz & 131 & 817 & 1.42 & 0 \\
\hline 9 & $\begin{array}{l}\text { Mehrabad - Mashhad - Dezfool - Shiraz - Ahvaz - } \\
\text { Isfahan - Bandarabas }\end{array}$ & 142 & 833 & 1.4 & 0 \\
\hline 10 & $\begin{array}{l}\text { Mehrabad - Mashhad - Dezfool - Shiraz - Ahvaz - } \\
\text { Isfahan - Yazd }\end{array}$ & 209 & 770 & 1.18 & 4 \\
\hline
\end{tabular}



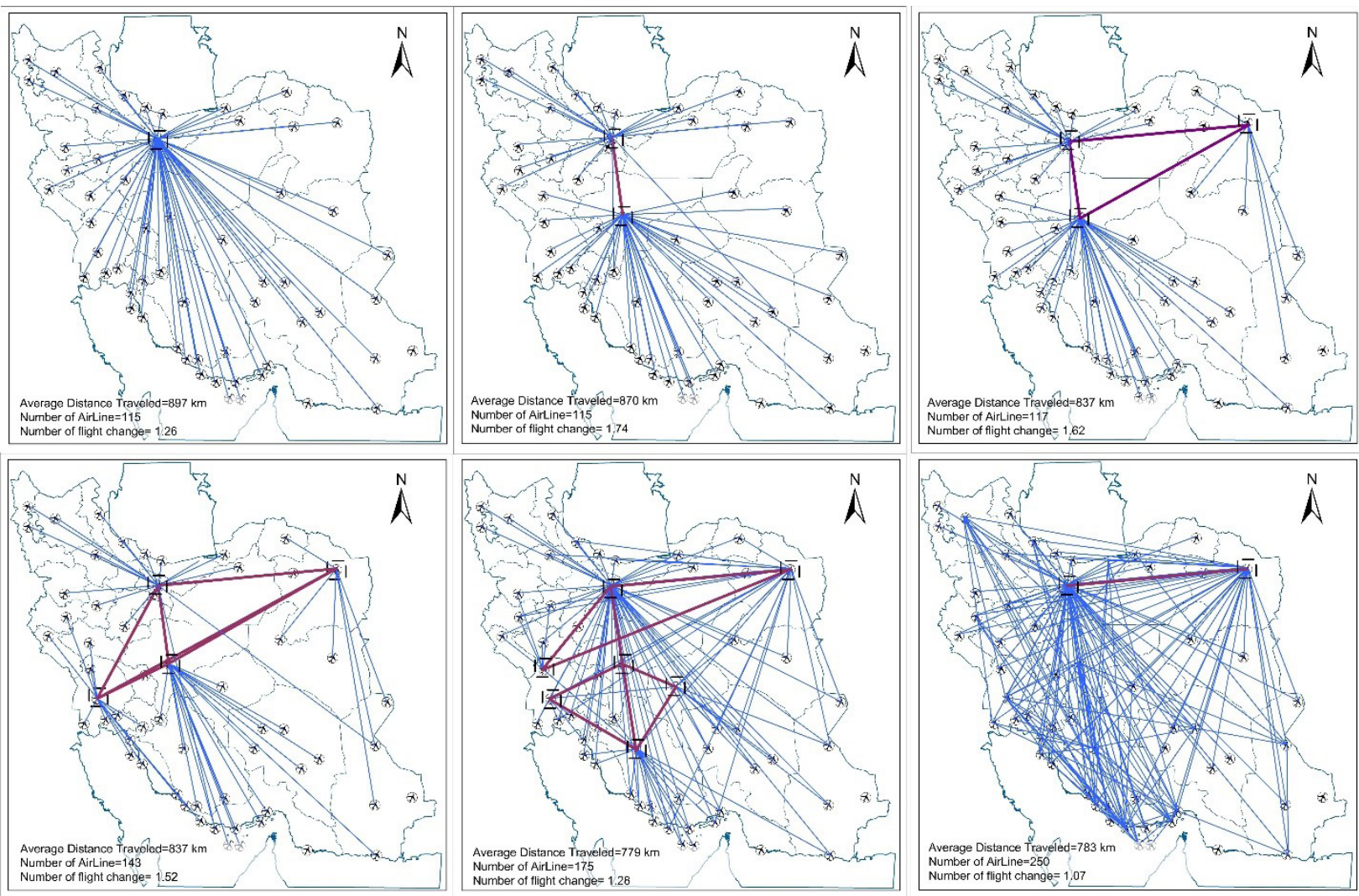

Fig. 7. Some of the airline architectures created by the proposed multi-object optimal topology.
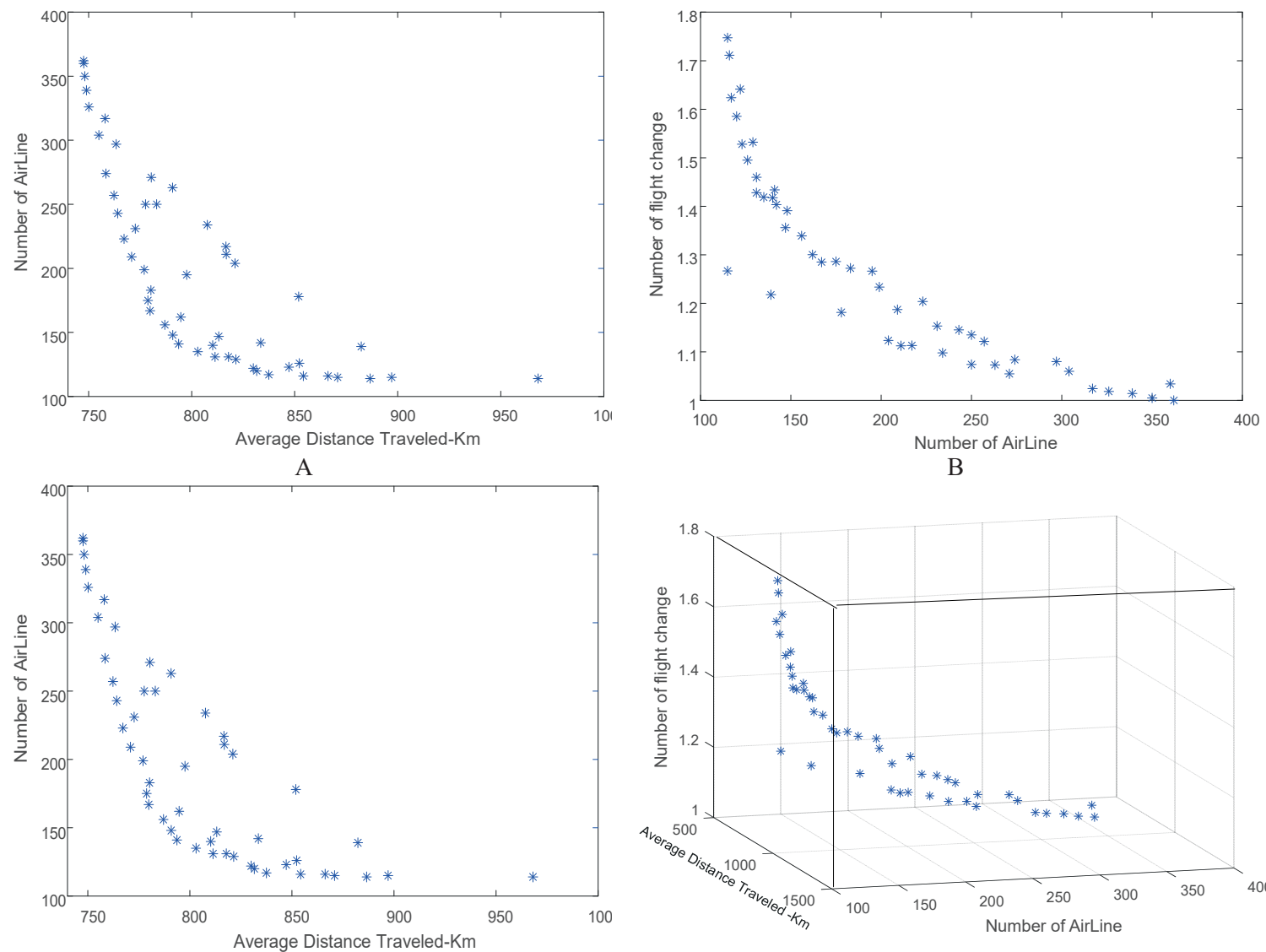

B

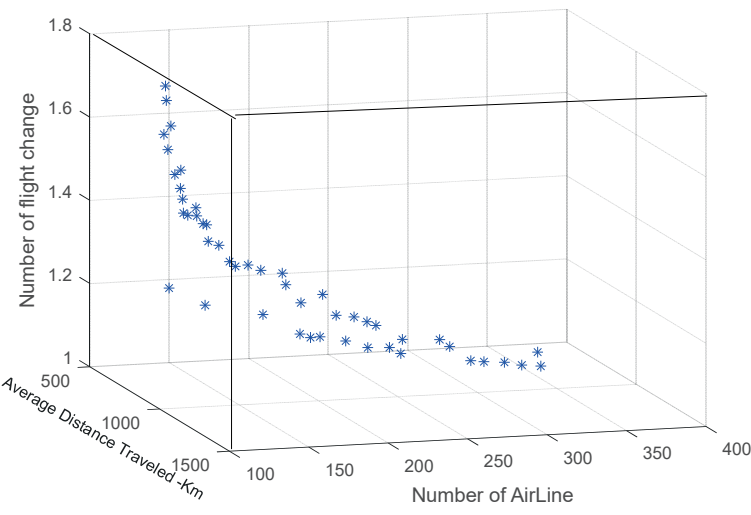

C

$\mathrm{D}$

Fig. 8. The optimal answers to the optimization airway network problem by the proposed model in three-dimensional space based on the value of target functions (Number of Airline vs. Number of flight change vs. Average travel length in Km). 
domination chart (the model of variation of the number of airline vs. number of flight change vs. average travel length in $\mathrm{Km}$ ) were used for assessing the proposed model. Convergence calculates the area covered by the answers to the parade (located in the target space) and path charts demonstrate the values of the optimal topology response set [29] in each of the target functions. The variations of the convergence plot of the proposed model for the supposed target functions have been normalized and can be seen in Fig. 9. This graph shows the qualitative evaluation of the obtained responses regarding each objective function.

As it is obvious in Fig. 9, the designed optimal model in this study has been able to improve the topology during different generations and move to optimal results. This matter suggests the possibility of discovering optimal answers in the designed model. Since the 300th generation so far, the ascending slope of the growing trend of the magnitude of the dispersion criterion has decreased, which indicates the convergence of the outputs of the proposed model to optimal solutions. The value chart of the solutions of the 500th generation achieved from the model is shown in Fig. 10. This figure was drawn for the normalized target functions.

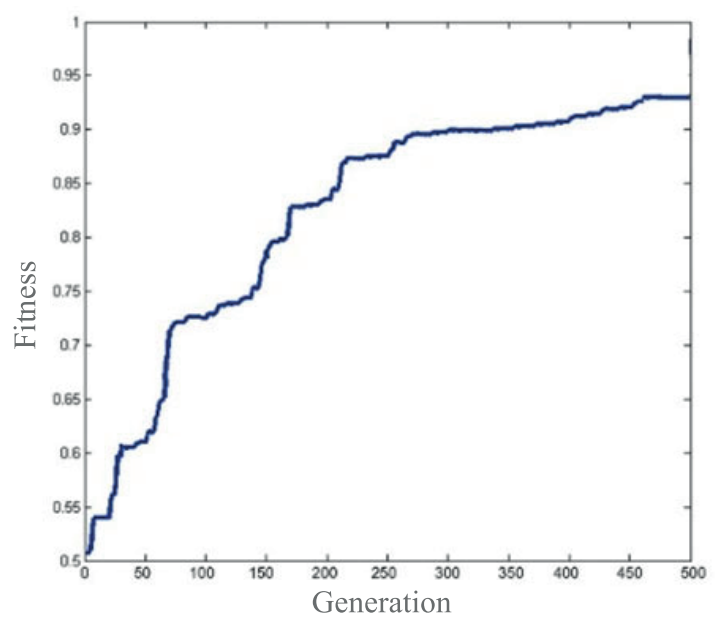

Fig. 9. The convergence plot of the proposed model

As it is shown in Fig. 10, the results obtained from the proposed model are expanded in each of the object functions throughout its target length, which indicates the acceptable dispersion of the results. Major changes are also observed in the slope of the routes between the two object functions, the number of airways and the average travel length, that suggest a good exchange between unsettled answers. On the other hand, significant changes in the gradient of the response slope are not observed in both functions - average travel length (target function \# 2) and average changes in routes (target function \# 3) - which indicates the presence of a correlation between these two target functions.

\section{B. Model Repeatability Test}

Since in multi-objective evolutionary algorithms, the search start point is chosen randomly, the results will vary with different performances. To test its repeatability, the model was performed four times with a population of 50 individuals with 300 repetitions with similar parameters. Fig. 11 shows the results from four times of repetition for the target function of the number of airways (Fig. 11-A) and average travel length (Fig. 11-B). As shown in the diagrams of this figure, the model has had a steady trend in all four repetitions, and the model outputs are almost located in a given range of target functions. The results are shown in Fig. 11 that compares the number of airways and average travel length, as two of the target functions, to present repeatability of the algorithm in the global optimization process. In the results of the four runs, there are similarities and differences between the number of airways and average travel length of the global solutions found based on the different selections of 50 individuals with 300 repetitions. From the investigation of the results, we found that the variance for the number of airways is in the range of $10 \%$, and it ranged between $5 \%$ for average travel length. From these results, it was concluded that the average repeatability of the proposed algorithm for different selections of individuals is about $93 \%$.

\section{CONCLUSIONS}

By studying Iran airlines, we understand that they can get more optimal. The proposed model outputs suggest some intermediate airports based on the Iran's aviation transport matrix. Therefore, there will be fewer airlines, and we can establish connections between airports with lesser money. Given the results of this paper, by considering a combination of point-to-point and Hub-and-spoke topologies, we would be able to offer more efficient airways. The designed model of this article tries to connect one airport to some intermediate airports,

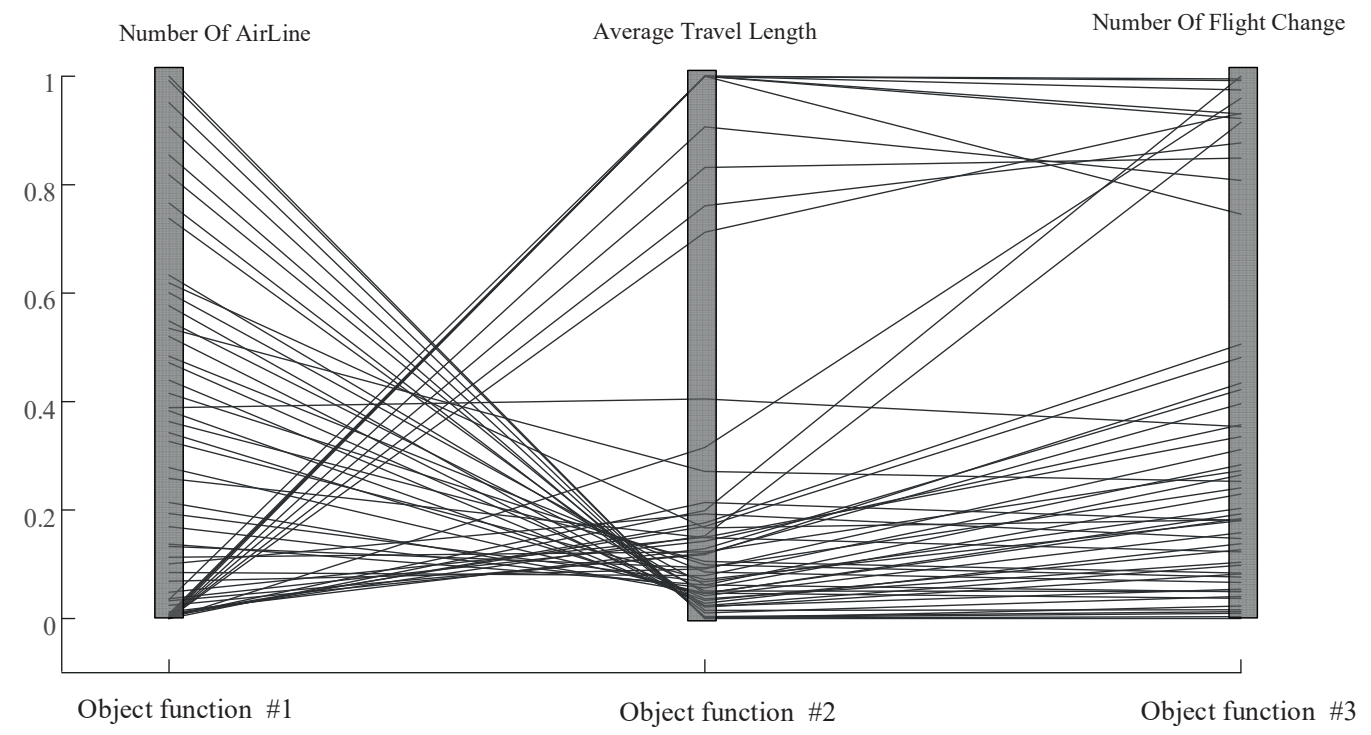

Fig. 10. The non-constrained-domination model (the model of variation of number of airways vs. number of flight change vs. average travel length in $\mathrm{Km}$ ) of the 500th generation. 


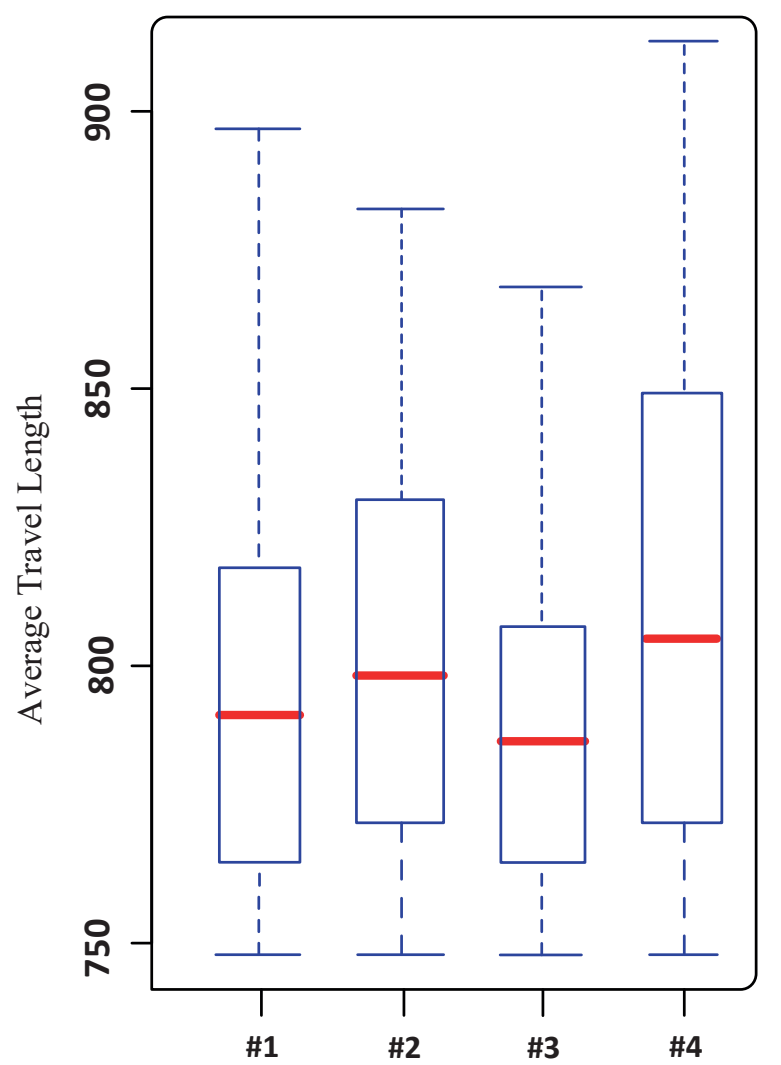

A

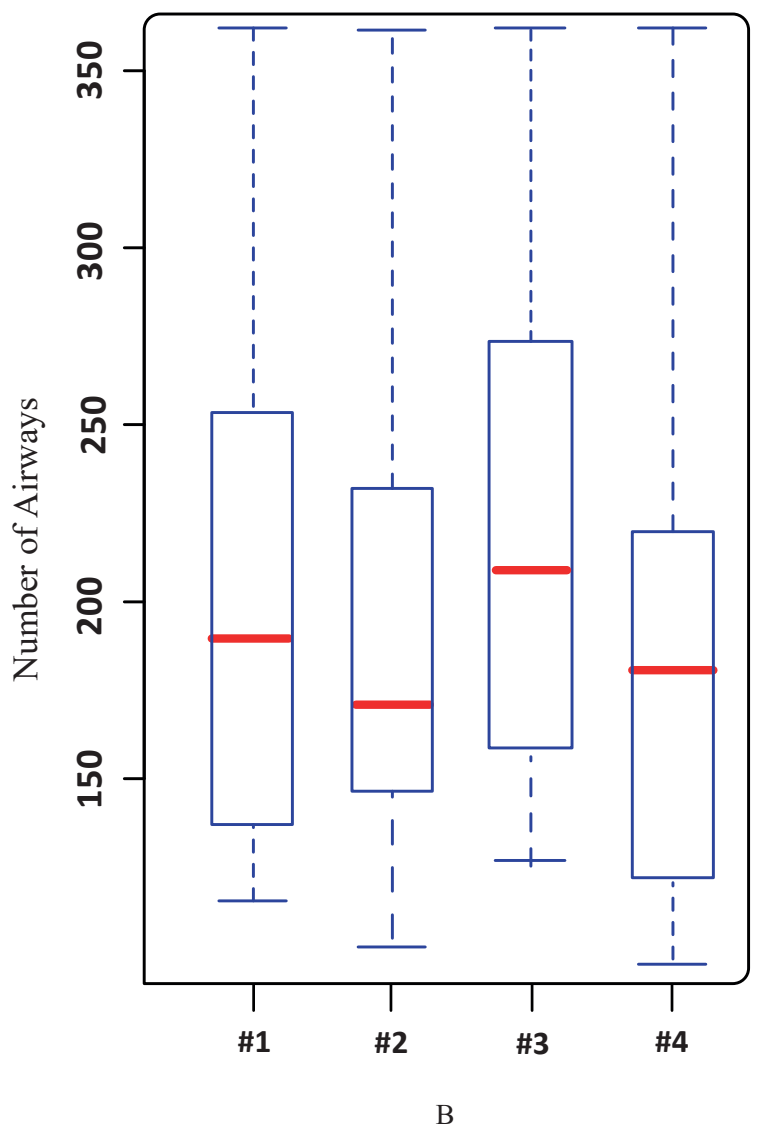

B

Fig. 11. Variations in the two target functions: average travel length (A), and the number of airways (B) and in different repetitions.

which are an excellent method to have more efficient airline topologies in the country. It should be noted that in airline topologies with the presence of one or more intermediate airports, besides having less populated airplanes and less expensive flights, we will have some economic benefits such as the ability to build convenience stores and economic prosperity in intermediate airports (such as Dubai Airport). We should remember that although airline topologies with intermediate airports increase changes in routes and therefore the travel length, with the aggregation of airways, the number of flights in an airway will increase and passengers could choose their flight hours during the day. One of the benefits of the designed model in this study is its capability in offering several options for airline topologies, and it lets managers and decision-makers select and apply one of the optimal topologies based on the current conditions. This feature also allows managers to replace another optimal topology if one or some intermediate airports are down because of whatever reason such as adverse weather conditions or something else. Scheduling flights in aviation topology design should be considered in future studies. So that the waiting time for a passenger in intermediate airports is viewed as a target function and efforts are focused on reducing it. Moreover, we should pay more attention to the capacity of air transport systems and airports and think of it as a limitation. Weather conditions as well as atmospheric environment matter while choosing an intermediate airport. It should be noted that, for some reason, such as policy domination or economic problems, airports that are not optimal will be viewed as intermediate airports. For instance, Dubai Airport is a convenient airport for the Middle East. Hence, it is crucial to consider the political and economic conditions in the selection of intermediate airports, which is recommended in future studies of this case too.

\section{REFERENCES}

[1] A. Fraile, J. Sicilia, R. González, and A. González, "Study of Factors Affecting the Choice Modal of Transportation in an Urban Environment Using Analytic Hierarchy Process," in Applied Computer Sciences in Engineering, Springer International Publishing, 2017, pp. 357-367.

[2] M. E. O'Kelly, "A quadratic integer program for the location of interacting hub facilities," European Journal of Operational Research, vol. 32, no. 3, pp. 393-404, 1987.

[3] P. Bagga, A. Joshi, and R. Hans, "QoS based Web Service Selection and Multi-Criteria Decision Making Methods," International Journal of Interactive Multimedia and Artificial Intelligence, vol. 5, no. 4, pp. 113$121,2019$.

[4] L. Mei, Z. Yan and J. Shao, "p-hub median location optimization of huband-spoke air transport networks in express enterprise," Special issue on multimodal information learning and analytics on cross-media big data, Concurrency and Computation: Practice and Experience, Wiley Online Library, vol. 31, no. 9, 2019.

[5] O. Lordan, J. M. Sallan and P. Simo, "Study of the topology and robustness of airline route networks from the complex network approach: a survey and research agenda," Journal of Transport Geography, vol. 37, pp. 112120, 2014.

[6] C. Liu and Q. Gui, "Mapping intellectual structures and dynamics of transport geography research: a scientometric overview from 1982 to 2014," Scientometrics, vol. 109, no. 1, p. 159-184, 2016.

[7] K. Deb, A. Pratap, S. Agarwal, and T. Meyarivan, "A fast and elitist multiobjective genetic algorithm: NSGA-II," IEEE Transactions on Evolutionary Computation, vol. 6, no. 2, pp. 182-197, 2002.

[8] S. Garcia-Rodriguez, "Application of Multiobjective Evolutionary Techniques for Robust Portfolio Optimization," International Journal of Interactive Multimedia and Artificial Intelligence, vol. 2, no. 2, pp. 63-64, 2013.

[9] M. Borhani and N. Ghasemloo, "Soft Computing Modelling of Urban Evolution: Tehran Metropolis," International Journal of Interactive 
Multimedia and Artificial Intelligence, p. In Press, 2020.

[10] A. H. A. Elkasem, S. Kamel, A. Rashad, and F. Jurado, "Optimal Performance of Doubly Fed Induction Generator Wind Farm Using Multi-Objective Genetic Algorithm," International Journal of Interactive Multimedia and Artificial Intelligence, vol. 5, no. 5, pp. 48-53, 2019.

[11] A. Taibi and B. Atmani, "Combining Fuzzy AHP with GIS and Decision Rules for Industrial Site Selection," International Journal of Interactive Multimedia and Artificial Intelligence, vol. 4, no. 6, pp. 60-69, 2017.

[12] M. Borhani, "Corpus Analysis Using Relaxed Conjugate Gradient Neural Network Training Algorithm," Neural Processing Letters, vol. 50, no. 1, p. 839-849, 2019.

[13] M. Borhani and H. Ghassemian, "Hyperspectral spatial-spectral feature classification based on adequate adaptive segmentation," in 2014 Iranian Conference on Intelligent Systems (ICIS), 2014.

[14] J. Sicilia, C. Quemada, B. Royo, and D. Escuín, "An optimization algorithm for solving the rich vehicle routing problem based on Variable Neighborhood Search and Tabu Search metaheuristics," Journal of Computational and Applied Mathematics, vol. 291, pp. 468-477, 2016.

[15] A. Fraile, E. Larrodé, A. Magreñan, and J. Sicilia, "Decision model for siting transport and logistic facilities in urban environments," Journal of Computational and Applied Mathematics, vol. 291, no. C, pp. 478-487, 2016.

[16] J. Sohn and S. Park, "A linear program for the two-hub location problem," European Journal of Operational Research, vol. 100, no. 3, pp. 617-622, 1997.

[17] V. Marianov and D. Serra, "Location models for airline hubs behaving as M/D/c queues," Computers \& Operations Research, vol. 30, no. 7, 983-1003.

[18] B. Wagner, "An exact solution procedure for a cluster hub location problem," European Journal of Operational Research, vol. 178, no. 2, p. 391-401, 2007.

[19] M. Mohammadi, F. Jolai and H. Rostami, "An M/M/c queue model for hub covering location problem," Mathematical and Computer Modelling, vol. 54, no. 11, pp. 2623-2638, 2011.

[20] S. A. Alumur, H. Yaman and B. Y. Kara, "Hierarchical multimodal hub location problem with time-definite deliveries," Transportation Research Part E: Logistics and Transportation Review, vol. 48, no. 6, pp. 11071120, 2012.

[21] F. Taghipourian, I. Mahdavi, N. Mahdavi-Amiri and A. Makui, "A fuzzy programming approach for dynamic virtual hub location problem," Applied Mathematical Modelling, vol. 36, no. 7, pp. 3257-3270, 2012.

[22] T. H. Yang and T. Y. Chiu, "Airline hub-and-spoke system design under stochastic demand and hub congestion," Journal of Industrial and Production Engineering, vol. 33, no. 2, pp. 69-76, 2016.

[23] P. K. Harshavardhan and K. Kousik, "Hierarchical Genetic Algorithms with evolving objective functions," Neural and Evolutionary Computing, p. arXiv:1812.10308, 2018.

[24] M. Khodemani-Yazdi, R. Tavakkoli-Moghaddam, M. Bashiri, and Y. Rahimi, "Solving a new bi-objective hierarchical hub location problem with an MMc queuing framework," Engineering Applications of Artificial Intelligence, vol. 78, pp. 53-70, 2019.

[25] D. L. Bryan. and M. E. O'Kelly, "Hub-and-Spoke Networks in Air Transportation: an Analytical Review," Journal of Regional Science, vol. 39, no. 2, pp. 275-295, 1999.

[26] S. W. C. Morrison, The economic effects of airline deregulation, Washington, DC.:Brookings Institution Press., 2010.

[27] D. E. Goldberg, Genetic algorithms in search, optimization, and machine learning, Boston, MA, USA: Addison-Wesley Longman Publishing Co., Inc., 1989.

[28] R. L. Haupt and S. E. Haupt, Practical genetic algorithms, John Wiley \& Sons, 2004.

[29] K. Deb, Multi-Objective Optimization Using Evolutionary Algorithms, New York, NY, USA: John Wiley \& Sons, Inc., 2001.

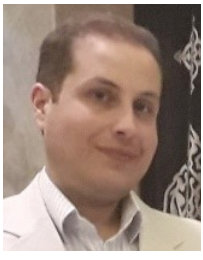

\section{Mostafa Borhani}

Dr. Mostafa Borhani is an expert at Communication System design, received the BSEE degree and MSEE degree from Sharif University of Technology, in 2002 and 2004, respectively and Ph.D. at the Department of Electrical and Computer Engineering, Tarbiat Modares University in 2015. He began his collaboration with high-tech PPPs and cooperated as a public-sector authority and advisors in some private-sector companies, time by time. He managed some national megaprojects such as DWDM national optical transport network (Tadbir) and IRIB Shima project. He is responsible as General Director of Administering and Leadership of OTT Service Operators in Cyberspace. His professional background includes scientific, research, and executive activities in advanced of structured networking, international relations, and the commercialization of knowledge based products. Artificial intelligence, computational intelligence, and data mining are some of his recent activities concerning extensive machine learning applications in sports, cyberspace and Holy Quranic data analysis to achieve more comprehension and understanding on this holy book. His research is conducted as faculty member of the Quran Miracle Research Institute of the Shahid Beheshti University.

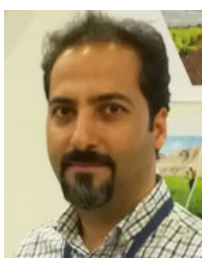

\section{Kamal Akbari}

Kamal Akbari is currently a researcher and spatial data analyst at GITA. He has over ten years of wide experiences in GIS, Spatial Database Designing, Pedestrian Modelling, and Teaching.

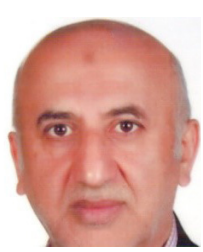

Aliakbar Matkan

Aliakbar Matkan is a Professor of Earth Sciences and Head of Remote Sensing and GIS Research Center, Faculty of Earth Sciences, Shahid Beheshti University.

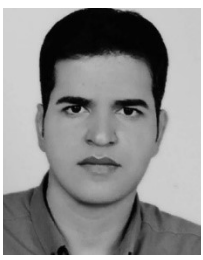

Mohammad Tanasan

Mohammad Tanasan is currently a Ph.D. Candidate in Remote Sensing and GIS center of Shahid Behehshti University. 